Tropical Journal of Pharmaceutical Research March 2016; 15 (3): 645-649

ISSN: $1596-5996$ (print); 1596-9827 (electronic)

(C) Pharmacotherapy Group, Faculty of Pharmacy, University of Benin, Benin City, 300001 Nigeria.

All rights reserved.

Available online at http://www.tjpr.org

Original Research Article

http://dx.doi.org/10.4314/tjpr.v15i3.29

\title{
Changes in Nitric Oxide Level and Thickness Index of Synovial Fluid in Osteoarthritis Patients following Intra- articular Injection of Sodium Hyaluronate
}

\author{
Laifeng $\mathrm{Li}^{1}$, Hui Wang ${ }^{1}$, Demin $\mathrm{Li}^{1}$ and Cunming Zhang ${ }^{2 \star}$ \\ ${ }^{1}$ The Third Hospital of Jinan, Jinan, Shandong, 250132, ${ }^{2}$ Department of Orthopedic Surgery, Shandong Provincial Qianfoshan \\ Hospital, Shandong, 250014, China
}

*For correspondence: Email: Ilf150609@163.com

Received: 28 August 2015

Revised accepted: 10 February 2016

\begin{abstract}
Purpose: To monitor the changes in nitric oxide levels and synovium thickness index in synovial fluid following intra-articular injection of sodium hyaluronate.

Methods: One hundred patients diagnosed with osteoarthritis of the knee from April 2014 to January 2015 in The Third Hospital of Jinan, Jinan, Shandong, China were selected and categorized into three phases; namely, mild, moderate and severe. Patients received a $20 \mathrm{~mL}$ sodium hyaluronate injection into the articular cavity of the knee once per week for 15 weeks, with continuous observation. The Visual Analogue Scale (VAS) and Western Ontario and McMaster University Osteoarthritis Index (WOMAC) scores were recorded after five weeks. A total of 56 patients (78 knees) remaining in serious condition after 5 weeks were divided into mild, moderate, and severe groups and treated with sodium hyaluronate once a week. Internationally reorganized VAS and WOMAC scores were adopted as clinical observation indices to indicate the curative effect of sodium hyaluronate among the 56 patients after 15 weeks of treatment. The conditions of the patients in the two phases were compared.

Results: After 5 weeks of treatment, treatment effective rate in the mild, moderate and severe groups was $72.92,66.10$ and $28.57 \%$, respectively, with an overall effective rate of $78 \%$. After 15 weeks of treatment, treatment effective rate in mild, moderate, and severe groups was $96.77,95.45$ and $66.67 \%$, respectively, with an overall effective rate of $67.95 \%$.

Conclusions: Clinically curative effect of sodium hyaluronate is significant for mild and moderate phase patients after intra-articular injection of sodium hyaluronate, while the effect is insignificant in severe patients. Thus, sodium hyaluronate can effectively improve nitric oxide levels in synovial fluid, reduce synovium thickness, enhances articular cavity lubrication and effectively alleviates disease severity.
\end{abstract}

Keywords: Osteoarthritis, Knee, Intra-articular injection, Sodium hyaluronate, Nitric oxide, Synovium thickness, WOMAC

Tropical Journal of Pharmaceutical Research is indexed by Science Citation Index (SciSearch), Scopus, International Pharmaceutical Abstract, Chemical Abstracts, Embase, Index Copernicus, EBSCO, African Index Medicus, JournalSeek, Journal Citation Reports/Science Edition, Directory of Open Access Journals (DOAJ), African Journal Online, Bioline International, Open-J-Gate and Pharmacy Abstracts

\section{INTRODUCTION}

Sodium hyaluronate is the major component of synovial fluid and one of the components of cartilage matrix. In patients with osteoarthritis, intra-articular injection of sodium hyaluronate can effectively improve articular cavity lubrication, covers and protects the cartilago articularis, improves joint contracture, inhibits degeneration changes on the cartilage surface, improves pathological joint fluid, and enhances articular cavity lubrication. With the aging population growing each year, the number of patients with knee osteoarthritis is increasing. Patients with 
knee osteoarthritis suffer from several detrimental symptoms, including knee joint pain, swelling, and limitation of activity.

Several groups have demonstrated that sodium hyaluronate has a lasting pain-relieving effect among patients with knee osteoarthritis, and this effect has been observed clinically. Chen et al [1] treated knee osteoarthritis by injecting sodium hyaluronate into the articular cavity of 484 patients (610 knees) and evaluated the effect of treatment using the Lysholm's knee score. The overall treatment success rate was $67.8 \%$. However, this effect was not observed among severely affected patients, and further work showed that a combination of surgery and drugs might be indicated for these patients [2].

Concurrent joint mobilization and intra-articular sodium hyaluronate injection may not only improve the effects of sodium hyaluronate treatment, but may also reduce pain and improve the effusion quantity and motion of joints in patients with knee osteoarthritis. Liu et al [3] treated patients with rheumatoid arthritis with oral NSAIDs and immunosuppressants with concurrent sodium hyaluronate injections of the knee joint. Although the treatment was generally successful, the onset of symptom relief was slow and of limited effect.

Nevertheless, local articular cavity treatment may control inflammatory responses and rapidly relieve joint pain. Therefore, $\mathrm{Ma}$ and Wang assessed the curative effect of applying Jintiange capsules together with sodium hyaluronate among 80 patients with knee osteoarthritis [4]. Using diagnostic criteria recommended by American College of Rheumatology, they confirmed that this treatment combination worked synergistically to relieve pain and repair cartilage.

\section{EXPERIMENTAL}

\section{Patient demographics}

One hundred patients in our hospital (121 knees) diagnosed with knee osteoarthritis from April 2014 to January 2015 in The Third Hospital of Jinan, Jinan, Shandong, China were selected for this study. Approval for the study was given by the institutional ethical committee. There were 54 males (62 knees) and 46 females (59 knees). Patients were grouped by disease severity after 5 weeks of treatment (Table 1) and re-evaluated after 15 weeks of treatment (Table 1).

\section{Therapeutic method}

For treatment with sodium hyaluronate, patients were seated with their knee joints fully exposed and at $90^{\circ}$ angle. lodine and ether were applied to the skin to sterilize the puncture point, and any synovial fluid present in the knee joint was extracted prior to the injection of $20 \mathrm{~mL}$ sodium hyaluronate. Injections were performed weekly for 15 weeks. The effect of treatment was evaluated via range of motion of the knee joint.

\section{Main outcome measures}

Measurement of NO levels in joint fluid: To examine nitric oxide (NO) levels in the joint fluid, $1 \mathrm{~mL}$ of fluid was extracted from the knee joint of each patient prior to treatment and each week of treatment. NO levels were determined nitrate reductase method and a spectrophotometer. NO levels were determined after 5 weeks and 15 weeks of treatment.

Ultrasonic testing of synovium thickness: GE LOGIQEQ ultrasound equipment (General Electric, Fairfield, CT, USA) together with the linear probe ML6-15 were used for ultrasonic inspection of knee join synovium thickness. Patients maintained their knee joints at a 30degree angle during the procedure, which was performed on transverse and vertical sections of the suprapatellar bursa. Synovium thickness was measure before treatment and after 5 and 15 weeks of treatment. All patients were examined by the same physician.

\section{Scoring format}

The Western Ontario and McMaster University Osteoarthritis Index (WOMAC) scoring standard was proposed and adopted clinically in 1988 [9]. This scoring system categorizes the severity of hip and knee arthritis and the curative effect of treatment in terms of some related symptoms and signs The Visual Analogue Scale (VAS) was used to record scores.

Table 1: Distribution of patients in disease severity groups after 15 weeks of treatment

\begin{tabular}{lll}
\hline Group & Male (32 cases, 43 knees) & Female (24 cases, 35 knees) \\
\hline Mild & 11 cases, 15 knees & 7 cases, 11 knees \\
Moderate & 18 cases, 23 knees & 15 cases, 20 knees \\
Severe & 3 cases, 5 knees & 2 cases, 4 knees \\
\hline
\end{tabular}


A straight line or ruler $10 \mathrm{~cm}$ in length is shown to patients, who mark the level of their pain or limited functional level on the line/ruler $(0=$ least severe, $10=$ most sever). The scoring index of WOMAC joint inflammation is shown in Table 2.

Table 2: WOMAC scoring index for joint inflammation

\section{Pain}

1. Walk on flat ground

2. Walk upstairs or downstairs

3. Pain that disturbs sleep on the bad at night

4. Sit or lie down

\section{Stiffness}

6. Severity of stiff after waking up in the morning

7. Severity of stiff after sitting, lying down and rest at a later period

\section{Difficulty in carrying out daily activities}

8. Walk downstairs

9. Walk upstairs

10. Stand up after sitting

11. Stand up

12. Bend towards the ground

13. Get in and out of the car or get on and off the bus

14. Shop outside

15. Wear socks or stockings

16. Get up from the bed

17. Take off socks or stockings

18. Lie in bed

19. Get in and out of the bath

20. While sitting

21. Squat down or stand up in toilet

22. Do heavy housework

23. Do light housework

Note: VAS is adopted to evaluate the index; a higher WOMAC index (score) indicates more severe osteoarthritis (OA). The degree of OA is determined by the total score: Mild < 80, moderate $=80-120$; severe $>120$

Table 3: Clinical outcome after 5 weeks of treatment

\section{Statistical analysis}

Data are presented as the mean \pm standard deviation. Differences between mean scores were tested using Student's t-test with SPSS 13.0 software. Differences were considered significant at $p<0.95$.

\section{RESULTS}

\section{Overall therapeutic effect of hyaluronate injection}

After 5 weeks of treatment, hyaluronate injections were effective in $72.92 \%$ of mild group, $66.10 \%$ of moderate group, and $28.57 \%$ of severe group patients with an overall effective rate of $78 \%$ (Table 3). After 15 weeks, treatment was effective in $96.77 \%$ of mild group, $95.45 \%$ of moderate groups, and $66.67 \%$ of severe group patients, with an overall effective rate of $67.95 \%$ (Table 4).

\section{Changes in synovial fluid NO levels}

Synovial fluid NO levels in patients with mild, moderate and severe knee osteoarthritis significantly improved after both 5 weeks and 15 weeks of treatment compared to baseline levels (before treatment; Table 5). Additionally, levels were significantly improved at 15 weeks of treatment compared to 5 weeks of treatment.

\section{WOMAC scoring}

As illustrated in Table 6, the effect of sodium hyaluronate treatment was significant by 5 weeks of treatment, and as shown in Table 7, although this effect was maintained through all 15 weeks of treatment, the effect was less significant in later weeks than during the first 5 weeks.

\begin{tabular}{|c|c|c|c|c|c|c|c|c|c|}
\hline \multirow{2}{*}{ Group } & \multicolumn{2}{|c|}{ No. of knees } & \multicolumn{2}{|c|}{$\begin{array}{c}\text { Significant } \\
\text { effect }\end{array}$} & \multicolumn{2}{|c|}{ Efficient } & \multicolumn{2}{|c|}{ Inefficient } & \multirow{2}{*}{ Effective rate } \\
\hline & $\begin{array}{c}\text { Male } \\
54\end{array}$ & $\begin{array}{c}\text { Female } \\
46\end{array}$ & Male & Female & Male & Female & Male & Female & \\
\hline Mild & 27 & 21 & 10 & 11 & 10 & 4 & 4 & 6 & $72.92 \%$ \\
\hline Moderate & 28 & 31 & 10 & 11 & 9 & 9 & 9 & 9 & $66.10 \%$ \\
\hline Severe & 7 & 7 & 1 & 0 & 2 & 1 & 4 & 4 & $28.57 \%$ \\
\hline
\end{tabular}

Table 4: Clinical outcome after 15 weeks of treatment

\begin{tabular}{lcccccccccc}
\hline \multirow{2}{*}{ Groups } & \multicolumn{2}{c}{ No. of knees } & \multicolumn{2}{c}{ Significant effect } & \multicolumn{2}{c}{ Efficient } & \multicolumn{2}{c}{ Inefficient } & \multirow{2}{*}{ Effective rate } \\
& Male & 43 Female & 35 & Male & Female & Male & Female & Male & Female & \\
\hline Mild & 16 & 15 & 7 & 10 & 8 & 5 & 1 & 0 & $96.77 \%$ \\
Moderate & 12 & 10 & 7 & 6 & 3 & 5 & 2 & 1 & $95.45 \%$ \\
Severe & 2 & 1 & 0 & 0 & 1 & 1 & 1 & 0 & $66.67 \%$ \\
\hline
\end{tabular}


Table 5: Synovial fluid NO levels $(\mu \mathrm{mol} / \mathrm{L})$ after 5 weeks of treatment

\begin{tabular}{lccccc}
\hline Group & No. of knees & Before treatment & 5 weeks later & t & $\boldsymbol{P}$-value \\
\hline Mild & 48 & $76.96 \pm 23.51$ & $50.29 \pm 20.12$ & 4.56 & 0.002 \\
Moderate & 59 & $93.73 \pm 48.29$ & $72.23 \pm 32.41$ & 4.69 & 0 \\
Severe & 14 & $145.34 \pm 68.32$ & $122.33 \pm 54.12$ & 4.72 & 0.001 \\
\hline
\end{tabular}

Table 6: Comparison of WOMAC Scores pre- and post (5 weeks) treatment

\begin{tabular}{lccc}
\hline Group & No. of knees & Before treatment & 5 weeks \\
\hline Mild & 48 & $77.58 \pm 10.38$ & $40 \pm 9.56$ \\
Moderate & 59 & $98 \pm 12.54$ & $51 \pm 11.25$ \\
Severe & 14 & $132 \pm 16.35$ & $116 \pm 12.68$ \\
\hline
\end{tabular}

Table 7: WOMAC scores after 5 and 15 weeks of treatment

\begin{tabular}{lccc}
\hline Group & No. of knees & 5 weeks & 15 weeks \\
\hline Mild & 31 & $73 \pm 10.16$ & $51 \pm 9.11$ \\
Moderate & 22 & $93 \pm 14.35$ & $77 \pm 9.86$ \\
Severe & 3 & $129 \pm 16.53$ & $119 \pm 14.25$ \\
\hline
\end{tabular}

\section{DISCUSSION}

Osteoarthritis is a chronic disease with a higher incidence rate among the elderly. The major clinical manifestations of the disease include pain, swelling, limitation of activity limitation, and clicking of the knee joint, which severely reduce the quality of life of patients. Osteoarthritis in the cartilage articularis first manifests as a progressive degeneration of the cartilage and reduction in sodium hyaluronate in the articular cavity, which results in the abnormal function of synovial fluid and leads to osteosclerosis of the subchondral bone and finally the formation of bone proliferation in the bond edge [5-7].

In this study, we demonstrated that the mechanism of action of sodium hyaluronate includes the improvement of joint fluid secretion, reduction in synovial fluid NO levels, and a decrease in synovium thickness. Additionally, injecting sodium hyaluronate into articular cavity may relieve inflammation, inhibit joint deterioration due to disease, and relieve pain, swelling, and activity limitation by promoting circulation in the joints and the recovery of knee joint function [8].

Nitrate concentrations were high in the joint fluid of knee osteoarthritis patients, and the inducible nitric oxide synthase inhibitor L-NMMA reduced the production of nitrates in tissue, indicating the production of $\mathrm{NO}$ in the joint fluid. Other groups have reported a serious imbalance in free radical metabolism in patients with knee osteoarthritis, resulting in widespread tissue damage in these patients. NO is a highly reactive free radical with a role in the regulation of blood pressure, nerve conduction, and host defense by killing bacteria, fungi, and cancer cells. Conversely, it can cause injury to normal tissues. Upon chondrocyte injury, the synthetic rate of nitric oxide synthase (cNOS)-and subsequently NO levels-increases $[10,11]$. NO is synthesized by inducible $\mathrm{NO}$ synthetase (iNOS), which can be activated by cytokines IL-1 $\beta$, TNF- $\alpha$, IFN- $\gamma$; therefore, NO may have a role in $r$ bone pathological process associated with cytokine activation.

The role of $\mathrm{NO}$ in the pathogenesis of knee osteoarthritis is gaining increased research attention. It has been found that iNOS inhibitors can effectively inhibit inflammatory lesions in the knee joint [12], improve local blood circulation, promote the cartilage repair, and reduce $\mathrm{NO}$ production in the knee joint, thus mitigating NOinduced damage to the cartilago articularis.

Sodium hyaluronate was first extracted from the bovine vitreous body in 1934 by Meyer et al [13], who described its role in pain suppression articular cartilage degeneration, the protection of articular tissue, the improvement of joint contracture and pathological joint fluid, and the promotion of the synthesis of more sodium hyaluronate in the synovial fluid. Sodium hyaluronate does not only prevent joint synovitis and cartilage degeneration, but it is also protective and reparative to degenerated synovial fluid and cartilage. The injection of sodium hyaluronate into the articular cavity has become an important prevention and complementary therapy for knee osteoarthritis [14]. Additionally, sodium hyaluronate can act to clear up cell debris generated from rapid metabolism in the joint. 
After intra-articular sodium hyaluronate injection, the treatment success rate was higher in patients with mild symptoms; the effective treatment rate in this group reached $72.92 \%$ after 5 weeks, while this rate was only $28.57 \%$ in the severe group. The treatment success rate further improved after 15 weeks of treatment compared to 5 weeks of treatment; however, effective of the treatment was still not apparent. Therefore, sodium hyaluronate treatment proved more effective in patients with mild disease than in those with severe disease. For patients with severe disease, surgery and/or other drugs are indicated.

We also observed a positive correlation between synovial fluid NO levels and clinical scores of knee osteoarthritis and synovium thickness. Additionally, NO levels were significantly reduced in all three disease groups (mild, moderate, and severe) after treatment with sodium hyaluronate. However, differences in synovium thickness were only significant in the moderate group, with no significant differences observed in the mild or severe group. This may indicate that knee articular cavity synovium in patients with mild osteoarthritis is close to normal levels and that sodium hyaluronate treatment relieves disease symptoms mainly by increasing articular synovial fluid metabolism [15]. However, as the synovium of patients with severe knee osteoarthritis is typically severely swollen, congested, full of lesions, sodium hyaluronate may be unable to penetrate the tissue, explaining the absent effect of treatment in patients with severe disease.

\section{CONCLUSION}

The findings of this study demonstrate that intraarticulate injection of sodium hyaluronate into the knee joint has a positive effect on articular cavity NO levels, promotion of joint fluid secretion, increased joint lubrication, and effective restoration of synovium thickness, thus confirming the mechanism of action of sodium hyaluronate.

\section{REFERENCES}

1. Chen M, Guo JF, Gu KM, Qin CR, Ren C, Chen GH. Curative effect of sodium hyaluronate injection in treating different stages of knee osteoarthritis. China Foreign Medical Treat 2014; 22: 110-112.
2. Liu Q. Clinical effect of joint mobilization combined with sodium hyaluronate in osteoarthritis treatment. China Modern Med 2015; 10: 81-83.

3. Liu D, Shen J, Chen QP, Cao TP, Wang ZL. 130 cases of injection of sodium hyaluronate in knee joint in rheumatoid arthritis treatment. Shaanxi Medical J, 2014; 3: 284-285.

4. Ma CZ, Wang XD. Clinical research of Jintiange capsule with sodium hyaluronate in knee osteoarthritis treatment. Shanxi Medical J, 2015; 7: 791-792.

5. Li FM, Zhao WH. Research on sodium hyaluronate combined with traditional Chinese medicine in treating knee osteoarthritis. Chinese J Trad Medical Traumatol Orthopaed, 2014; 9: 79-80.

6. Zhu BJ, Chen ZF, Liu F, etc. The safety and efficacy of simultaneous or staged bilateral total knee arthroplasty in treatment of both knee osteoarthritis. Chinese $J$ Orthopaed, 2014; (6): 672-675.

7. Gao SG, Xu WS, Zeng KB, etc. Correlation of knee osteoarthritis joint synovial fluid and osteopontin level with lesion degree. Chinese J Orthopaed, 2010; 7: 672675.

8. Liao CM, Jiang YL, Liao W. Curative observation of articular injection of sodium hyaluronate in treating 48 cases of osteoarthritis. Modern Med Health, 2014; 1: 2498-2500.

9. Shu H. Observation on effect of articular injection of sodium hyaluronate combined with warm needle in treating knee osteoarthritis. World Fed Chinese Med, 2014; 11: 1528-1530.

10. Deng JF, Gao DX, Liu ZX. Effect of sodium hyaluronate on nitric oxide and synovial thickness in patients with knee osteoarthritis. J Clin Rehab Tissue Eng Res, 2014; 52: 8520-8524.

11. Mototani $H$, lida A, Nakajima M. A functional SNP in EDG2 increases susceptibility to knee osteoarthritis in Japanese. Hum Mol Genet. 2008; 17(12): 1790-1797.

12. Han DM, Gao CY, Zhang YH, Zhao J. The expression of $N O$ in cartilage degeneration and synovial tissue in patient with osteoarthritis. Chinese $J$ Biochem Pharmaceut, 2011; 3: 245-248.

13. Liu ZP, Wang B. New clinical applications of hyaluronic acid. Food Drug A, 2006; 12: 8-10.

14. Wang GX, Zheng CY, Li L, Fang W. Clinical effect of articular cavity injection of sodium hyaluronate in treating knee osteoarthritis. J Nurs Training, 2010; 9: 832-833.

15. Wang BJ, Wang ZF. The effect of Chubi lotion to the free radical level of knee osteoarthritis joint fluid and $I L-1 \beta$ and TNF- $\alpha$. J Beijing Trad Chinese Med, 2011; 10: 737739. 